\title{
Factors associated with subsequent surgical procedures after intramedullary nailing for tibial shaft fractures
}

\author{
Laurent A. M. Hendrickx ${ }^{1,2} \oplus$. James Virgin ${ }^{1} \cdot$ Job N. Doornberg ${ }^{1,2} \cdot$ Gino M. M. J. Kerkhoffs ${ }^{2} \cdot$ Ruurd L. Jaarsma $^{1}$
}

Received: 23 March 2020 / Accepted: 1 July 2020 / Published online: 12 July 2020

(c) The Author(s) 2020

\begin{abstract}
Introduction The reported rate of subsequent surgery after intramedullary nailing (IMN) of tibial shaft fractures (TSFs) is as high as $21 \%$. However, most studies have not included the removal of symptomatic implant in these rates. The purpose of this study was to evaluate the subsequent surgery rate after IMN of TSFs, including the removal of symptomatic implants. Secondly, this study aimed to assess what factors are associated with subsequent surgery (1) to promote fracture and wound healing and (2) for the removal of symptomatic implants.

Methods One-hundred and ninety-one patients treated with IMN for TSFs were retrospectively included. The rate of subsequent surgery was determined. Bi- and multivariable analysis was used to identify variables associated with subsequent surgery.

Results Approximately half of patients (46\%) underwent at least one subsequent surgical procedure. Forty-eight $(25 \%)$ underwent a subsequent surgical procedure to promote fracture or wound healing. Age $(P<0.01)$, multi-trauma $(P<0.01)$, open fracture $(P<0.001)$ and index surgery during weekdays $(P<0.05)$ were associated with these procedures. Thirty-nine patients $(20 \%)$ underwent a subsequent surgical procedure for removal of symptomatic implants. There was a significantly lower rate of implant removal in ASA II (11\%) and ASA III-IV (14\%) patients compared to ASA I patients $(29 \%)(P<0.05)$. Conclusions Patients treated with IMN for TSFs should be consented that about one-in-two patients will undergo an additional surgical procedure. Half of these procedures are required to promote wound or fracture healing; the other half are for symptomatic implant removal.
\end{abstract}

Level of evidence Therapeutic level-IV.

Keywords Tibial shaft fracture $\cdot$ Subsequent surgery $\cdot$ Predictors $\cdot$ Implant removal $\cdot$ Intramedullary nailing

\section{Introduction}

Tibial shaft fractures (TSFs) are frequently occurring injuries [1]. Intramedullary nailing (IMN) is widely considered the best treatment for these injuries because it provides good direct relative fracture stability whilst being minimally invasive with regard to surrounding soft tissue [2]. Nevertheless, for many patients IMN is only the first operation in

Laurent A. M. Hendrickx ranhendrickx@gmail.com

1 Department of Orthopaedics and Trauma Surgery, Flinders Medical Centre, Flinders University, Adelaide, SA 5042, Australia

2 Department of Orthopaedic Surgery, Amsterdam UMC, University of Amsterdam, Amsterdam Movement Sciences, Amsterdam, The Netherlands the process of achieving satisfactory operative outcomes, with (several) additional surgical procedures often required. Current literature reports on reoperation rates after operative treatment of TSFs ranging from 14 to $36 \%$; however, few studies have directly investigated this study question (Table 1) [3-6]. Furthermore, the majority of these studies do not include or report on removal of implant due to local pain or irritation as a secondary procedure as these are considered discretionary. In a recent review of the literature, we found the average rate of symptomatic screw removal after IMN of TSFs to be 9\% [7-12]. However, from the experience at our level-1 trauma centre we believe this to be an underestimation, hypothesizing that the true rate of screw removal, and therefore the true rate of subsequent surgery, is significantly higher.

Surgery for the removal of symptomatic implants can have a significant impact at a socio-economic level [13] and 
Table 1 Previous studies investigating factors associated with subsequent surgery after operative treatment of tibial shaft fractures

\begin{tabular}{|c|c|c|c|c|}
\hline Authors & Patients & Minimum follow-up & Subsequent surgery rate & $\begin{array}{l}\text { Factors associated with subsequent } \\
\text { surgery }\end{array}$ \\
\hline Stavrou et al. [3] & 151 treated with IMN & 12 months & $21 \%$ & $\begin{array}{l}42 \mathrm{~B} \text { or } 42 \mathrm{C} \mathrm{AO} / \mathrm{OTA} \text { fracture type } \\
\text { Alcohol abuse }\end{array}$ \\
\hline Fong et al. [4] & $\begin{array}{l}157 \text { treated with IMN } \\
36 \text { treated with plate fixation }\end{array}$ & Unclear & $13.5 \%$ overall & $\begin{array}{l}\text { Open fractures } \\
\text { Transverse fractures }\end{array}$ \\
\hline Bhandari et al. [5] & $\begin{array}{l}80 \text { treated with IMN } \\
108 \text { treated with plate fixation } \\
4 \text { treated with external fixator }\end{array}$ & 12 months & $\begin{array}{l}16.3 \% \text { for IMN } \\
22.4 \% \text { overall }\end{array}$ & $\begin{array}{l}\text { Open fractures } \\
\text { Cortical contact }<50 \% \\
\text { Transverse fractures }\end{array}$ \\
\hline Harris and Lyons [6] & $\begin{array}{l}124 \text { treated with IMN } \\
17 \text { treated with external fixator } \\
1 \text { treated with plate fixation }\end{array}$ & 6 months & $35.8 \%$ overall & $\begin{array}{l}\text { 42B AO/OTA fracture type } \\
\text { Gustilo-Anderson Grade II and III }\end{array}$ \\
\hline
\end{tabular}

can increase the risk of additional complications [13, 14]. Decreasing the rate of these procedures should therefore be considered an important goal. However, to the best of our knowledge, thus far, no factors associated with implant removal have been identified.

The primary aim of this study was to assess the total rate of subsequent surgery after IMN of TSFs, including symptomatic implant removals. The secondary aim was to assess what patient, trauma and fracture characteristics are associated with (1) subsequent surgery for wound and fracture healing and (2) subsequent surgery for implant removal. This knowledge will allow clinicians to better inform patients on expected outcomes following surgery. Additionally, it will allow for better insight into the total health economic costs associated with IMN of TSFs.

\section{Materials and methods}

\section{Ethics}

In accordance with the Declaration of Helsinki, our institutional review board granted approval for this study (Reference number: AUD/19/SAC/250).

\section{Study design, setting and participants}

As per protocol, all TSFs at our level-1 trauma centre are treated with reamed IMN with the TRIGEN Intramedullary Nailing System (Smith \& Nephew, Andover, MA USA) with proximal and distal interlocking screws. Postoperatively, patients were allowed to weight bear as tolerated. Patients were routinely seen at 2, 6 and 12 weeks after surgery, or longer in case of an atypical recovery. Implant removal was not part of the standard treatment.
We included all skeletally mature patients with traumatic TSFs who were treated with IMN between January 2009 and September 2016, allowing for a minimum follow-up of 2.5 years. Patients treated for pathological fractures, patients with incomplete records and patients with inadequate follow-up (i.e. $<12$ weeks) were excluded.

\section{Variables, outcome measures, data sources and bias}

Two authors not involved in patient care (LH and JV) assessed radiographs, patients' files, operation reports and CT scans to collect patient, trauma, fracture and treatment characteristics.

Independent variables included: (1) gender; (2) age; (3) multi-trauma; (4) trauma mechanism; (5) American Society of Anesthesiologists Physical Status (ASA-PS) [15]; (6) open fracture; (7) OTA/AO type of tibial fracture; (8) location of tibial fracture; (9) presence of fibula fracture; (10) the use $>2$ proximal screws; (11) the use $>2$ distal screws; (12) surgery during weekend or weekday; (13) after-hours surgery; and (14) level of surgeon. Fractures were classified into three groups according to the OTA/AO Fracture and Dislocation Classification Compendium: 42A1-3, 42B1-3 and 42C1-3. Trauma mechanism was classified as either low energy ( $<30 \mathrm{~km}$ per hour or a fall from $<3 \mathrm{~m}$ ) or high energy. After-hours surgery was defined as any surgery starting between $18.00 \mathrm{pm}$ and $08.00 \mathrm{am}$. The cut-off for $>2$ proximal or distal interlocking screws was chosen because, from our experience, the use of more locking screws is usually related to surgery for more complex fractures.

The primary outcome of this study was subsequent surgery, including elective procedures. It was recorded whether patients underwent one, two or more than two subsequent surgical procedures. Subsequent surgical procedures were also categorized into the following: (1) subsequent surgery 
to promote union (dynamization, nail exchange, bone graft); (2) wound closure (delayed primary wound closure, skin graft, flap or closure of fasciotomy wounds); (3) fasciotomies for postoperative compartment syndrome; (4) surgery to treat infection; (5) surgery to correct malunion or rotational malalignment; (6) surgery for wound healing (washout and debridement); (7) removal of interlocking screw due to irritation or pain; and (8) removal of tibial nail due to pain or irritation. Using these eight categories, two main groups were distinguished: (1) patients with a subsequent surgical procedure for fracture and wound healing (categories 1-6) and (2) and patients with a subsequent surgical procedure to remove symptomatic screws and/or tibial nail (categories 7 and 8$)$.

\section{Statistical analysis}

Qualitative assessment of the data was performed. Descriptive statistics were calculated: means and standard deviations for normally distributed continuous variables, median and range for non-normally distributed continuous variables and frequencies and percentages for categorical variables.

Bivariable analysis was performed to assess whether any independent variables were associated with each respective group of subsequent surgical procedures. Binary logistic regression was used for continuous variables, and $\chi^{2}$ test or Fisher exact was used for categorical and ordinal variables. Variables with a $P$ value $<0.1$ were subsequently entered in a multivariable binary logistic regression with a stepwise backward selection procedure. At each step, the variable with the largest $P$ value was eliminated. This process was repeated until all variables in the equation reached a $P$ value $<0.05$. Multivariable binary logistic regression was limited to five events per variable.

Regarding subsequent surgery for symptomatic screws, we performed a subgroup analysis of patients who had undergone protocolled low-dose postoperative bilateral CT scans for the assessment of rotational malalignment [16]. This protocol was implemented at our institution in 2009 with an initial adherence rate of $43 \%$. In 2018, the adherence rate of this protocol had increased to $83 \%$. In a previous study, we analysed these postoperative CT scans to assess the incidence of iatrogenic screw penetration in the proximal and distal tibiofibular joint [17]. In the current study, we reused these data, to assess whether these types of screw penetration are associated with a higher rate of symptomatic screw removal.

\section{Results}

From 2009 to 2016, 251 patients were treated with IMN for TSFs. Sixty patients (24\%) were excluded: 36 patients (14\%) had inadequate follow-up, 21 patients $(8 \%)$ were followed up externally, one patient received palliative care after surgery, one patient had incomplete records and one patient had a pathological fracture.

A total of 191 patients were included. The majority of patients were male $(71.2 \%)$ with a median age of 37 years (range, 14-90 years). Eighty patients (42\%) sustained the fracture in a high-energy trauma, and $39(20 \%)$ were polytrauma patients. Further patient and fracture characteristics are displayed in Table 2.

Eighty-seven patients (46\%) underwent at least one subsequent surgical procedure. The most frequent indication for a first subsequent surgical procedure was screw removal due to irritation or pain (40\%), followed by closure of wounds (25\%) (Table 3). Twenty-nine patients (15\%) underwent at least two subsequent surgical procedures. The most frequent second additional surgical procedures were performed for wound healing (31\%), followed by closure of wounds (21\%) (Table 3). Thirteen patients (7\%) underwent more than two additional surgical procedures.

\section{Subsequent surgery fracture and wound healing}

Forty-eight patients (25\%) underwent a first subsequent surgical procedure to promote fracture or wound healing. Bivariable analysis demonstrated that age $(P<0.05)$, multi-trauma $(P<0.001)$, trauma-mechanism $(P<0.001)$, open fracture $(P<0.01)$, AO/OTA type $(P<0.01)$, the use of more than 2 proximal interlocking screws $(P<0.05)$ and surgery during weekdays $(P<0.05)$ were associated with subsequent surgical procedures for fracture and wound healing (Table 4).

Multivariable analysis subsequently identified younger age $(P<0.01)$, multi-trauma $(P<0.01)$, open fracture $(P<0.001)$ and surgery during weekdays $(P<0.05)$ as independent predictors (Table 5).

\section{Subsequent surgery for removal of symptomatic screws and/or nail}

Removal of symptomatic screws and/or nail occurred on average 578 days after the index procedure (range 94-1850 days). Thirty-nine patients (20\%) underwent a first subsequent surgical procedure for removal of symptomatic screws or nails. Bivariable analysis indicated that only ASA-PS was associated with this type of subsequent surgery (Table 6). The rate of implant removal was significantly 
Table 2 Patient demographics and fracture characteristics $(n=191)$

\begin{tabular}{|c|c|}
\hline \multicolumn{2}{|l|}{ Patient characteristics } \\
\hline Age, median years (range) & $37(14-90)$ \\
\hline \multicolumn{2}{|l|}{ Gender, $n(\%)$} \\
\hline Male & $55(29 \%)$ \\
\hline Female & $136(71 \%)$ \\
\hline \multicolumn{2}{|l|}{ Multi-trauma, $n(\%)$} \\
\hline No & $152(80 \%)$ \\
\hline Yes & $39(20 \%)$ \\
\hline \multicolumn{2}{|l|}{ Trauma mechanism, $n(\%)$} \\
\hline Low energy & $111(58 \%)$ \\
\hline High energy & $80(42 \%)$ \\
\hline \multicolumn{2}{|l|}{ ASA-PS, $n(\%)$} \\
\hline ASA I & $92(48 \%)$ \\
\hline ASA II & $64(34 \%)$ \\
\hline ASA III-IV & $35(18 \%)$ \\
\hline \multicolumn{2}{|l|}{ Fracture characteristics } \\
\hline \multicolumn{2}{|l|}{ Open fracture, $n(\%)$} \\
\hline No & $128(67 \%)$ \\
\hline Yes & $63(33 \%)$ \\
\hline \multicolumn{2}{|l|}{ AO/OTA type, $n(\%)$} \\
\hline $42 \mathrm{~A} 1-3$ & $119(62 \%)$ \\
\hline $42 \mathrm{~B} 1-3$ & $44(23 \%)$ \\
\hline $42 \mathrm{C} 1-3$ & $28(15 \%)$ \\
\hline \multicolumn{2}{|l|}{ Location, $n(\%)$} \\
\hline Proximal & $6(3 \%)$ \\
\hline Middle & $58(30 \%)$ \\
\hline Distal & $116(61 \%)$ \\
\hline Segmental & $11(6 \%)$ \\
\hline \multicolumn{2}{|l|}{ Fibula fracture, $n(\%)$} \\
\hline No & $27(14 \%)$ \\
\hline Yes & $164(86 \%)$ \\
\hline \multicolumn{2}{|l|}{ Surgery characteristics } \\
\hline \multicolumn{2}{|l|}{$>2$ proximal screws, $n(\%)$} \\
\hline No & $175(92 \%)$ \\
\hline Yes & $16(8 \%)$ \\
\hline \multicolumn{2}{|l|}{$>2$ distal screws, $n(\%)$} \\
\hline No & $145(76 \%)$ \\
\hline Yes & $46(24 \%)$ \\
\hline \multicolumn{2}{|l|}{ Day of operation, $n(\%)$} \\
\hline Weekday & $127(66 \%)$ \\
\hline Weekend & $64(34 \%)$ \\
\hline \multicolumn{2}{|l|}{ After-hours surgery, $n(\%)$} \\
\hline No & $145(76 \%)$ \\
\hline Yes & $46(24 \%)$ \\
\hline \multicolumn{2}{|l|}{ Level primary surgeon, $n(\%)$} \\
\hline Consultant & $71(37 \%)$ \\
\hline Fellow & $68(36 \%)$ \\
\hline Registrar & $52(27 \%)$ \\
\hline
\end{tabular}

lower in ASA II and ASA III-IV patients as compared to ASA I patients $(P<0.05)$.

\section{Subgroup analysis of screw penetration}

A total of 123 patients had undergone a low-dose postoperative CT scan to assess malalignment according to hospital protocol. Of these patients, 18 were excluded; in three patients, it was unclear which screw had been removed since no follow-up radiology was available; three patients had undergone dynamization to promote union; and in 12 patients, the tibial nail had been revised, removed or exchanged after the CT scan. In the remaining 105 patients, no association between proximal or distal tibiofibular screw penetration and screw removal could be demonstrated (Table 7).

\section{Discussion}

Patients treated with IMN for TSFs should be consented that about one-in-two patients will undergo an additional surgical procedure. Approximately half of these additional surgical procedures are performed to promote fracture or wound healing. Age, multi-trauma, open fractures and index surgery during weekdays are predictors of this type of additional surgical procedures. The other half of procedures are discretionary: performed to remove interlocking screws and/or tibial nails causing pain or irritation. This type of procedures is less frequently performed in patients with higher ASA-PS and is not associated with tibiofibular screw penetration. These data support the consent of patients with TSFs: that IMN may not be a quick fix.

The findings of this study must be appreciated with an understanding of its limitations. Firstly, a substantial number $(22 \%)$ of patients had to be excluded due to inadequate follow-up. Although loss to follow-up is a well-known problem in Orthopaedic Trauma [18]; in the current study, this relative high number was partly caused by $8 \%$ of patients being followed up externally. This is common practice at our hospital, as our hospital services rural locations more than $1000 \mathrm{~km}$ away. Secondly, this study was conducted retrospectively. One of the disadvantages of this design is that the independent variables tested were limited to those that had been collected previously. Potentially important variables such as alcohol abuse [3] could therefore not be included. Thirdly, this study was conducted at a single, level-1 trauma centre. This may have resulted in a slight overrepresentation of high-energy trauma and open fractures. However, since mono-trauma cases are also part of 
Table 3 Overview of the first and second additional subsequent surgical procedures patients underwent

\begin{tabular}{lcc}
\hline Type of subsequent surgical procedure & $\begin{array}{l}\text { First subsequent surgical } \\
\text { procedure, } n(\%)\end{array}$ & $\begin{array}{l}\text { Second subsequent sur- } \\
\text { gical procedure, } n(\%)\end{array}$ \\
\hline Surgery to promote union & $9(10 \%)$ & $3(10 \%)$ \\
Surgery to close wounds & $22(25 \%)$ & $6(21 \%)$ \\
Fasciotomy postoperative compartment syndrome & $4(5 \%)$ & $2(7 \%)$ \\
Surgery to treat infection & $2(2 \%)$ & $1(3 \%)$ \\
Surgery to correct malunion & $5(6 \%)$ & $3(10 \%)$ \\
Surgery to promote wound healing & $6(7 \%)$ & $9(31 \%)$ \\
Removal symptomatic screw & $35(40 \%)$ & $5(17 \%)$ \\
Removal symptomatic nail & $4(5 \%)$ & $5(17 \%)$ \\
Total & $87(100 \%)$ & $29(100 \%)$ \\
\hline
\end{tabular}

our daily routine practice and represented $80 \%$ of the entire cohort, we believe the current series is a good representation of the entire spectrum of tibial shaft fractures. Lastly, limited by the number of events per variable we were forced to group a number of independent variables. This may have concealed the effect of certain variables such as the previously documented effect of transverse fractures on the reoperation rate $[4,5]$.

The one-in-two reoperation rate $(46 \%)$ identified in this study is substantially higher than previously reported (14-36\%) [3-6]. This is mainly due to the large number $(n=39)$ of surgical procedures carried out for symptomatic screw removal, which is not included in the majority of the previously reported studies, but very important in informed consent for our patients in the overall picture. It could be argued that the removal of symptomatic locking screws is a relatively minor surgical procedure; however, from a patients' perspective any type of surgery is often subjectively considered as major. With an estimated total procedural cost of \$2000-2500 (AUD) at our institution, this type of surgery can furthermore have significant impact at a socio-economic level [13]. We therefore believe that it is important for clinicians and patients to be aware of this substantial number. It is important to note that the high rate of implant removal is not exclusive to IMN. In a randomized controlled trial comparing IMN and plate fixation of distal TSFs, the rate of subsequent surgery for implant removal was similar between both groups [19].

It is well known that open TSFs are at a higher risk of infection and non-union [20-23]. In the current study, open fractures were identified as an independent predictor of subsequent surgery. This is in line with what several previous studies have demonstrated [4-6] (Table 1). Both younger age and multi-trauma were also predictive of subsequent surgery for wound and fracture healing. Both of these variables may be considered indicative of injury severity. With regard to age, this can be explained by the bimodal distribution of TSFs: in younger patients, they are more often caused by traffic accidents, whereas in elderly patients they are most commonly caused by simple falls [24]. Lastly, surgery on weekdays was an independent predictor of subsequent surgery for wound and fracture healing. When initiating this study, we hypothesized the opposite to be true, as various studies have suggested outcome may be worse if patients are admitted or undergo surgery during the weekend [25-27]. A possible explanation for our finding could be that there may be a tendency to postpone non-acute, yet complex cases during the weekend to weekdays.

Only one variable was associated with subsequent discretionary surgery to remove symptomatic screws and/or nails: in patients with higher ASA-PS significantly less surgery was performed to remove implant. This is likely explained by surgeons and anaesthesiologists being more cautious with additional surgery in this patient group, rather than there being a causal relationship between ASA-PS and symptomatic screws. On the other hand, it may also indicate that we need to more critically review whether removal of screws and/or nails in patients with an ASA I status is necessary as one could argue that this is elective. Whilst it is suggested that screw penetration in the proximal and distal tibiofibular joint may lead to respective lateral sided kneepain [17, 28] and lateral sided ankle pain [17], there was no higher rate of removal of these screws in our study. Future studies should aim to assess whether screw penetration in the proximal or distal tibiofibular joint indeed causes pain or affects functional outcome. It is important to note that the interlocking screws which were used in this study have been modified in order to give the screw heads a lower profile. This modification was introduced in our hospital after our final inclusion. Future studies should be performed to assess whether this modification results in lower rates of screw removal. 
Table 4 Bivariable Analysis of Patient, Trauma, Fracture and Treatment Characteristics and Subsequent Surgery for Fracture and Wound Healing $(n=191)$

\begin{tabular}{|c|c|c|c|}
\hline \multirow[t]{2}{*}{ Variable } & \multicolumn{2}{|c|}{$\begin{array}{l}\text { Subsequent surgery for } \\
\text { fracture and wound healing }\end{array}$} & \multirow[t]{2}{*}{$P$ value } \\
\hline & No & Yes & \\
\hline Gender, $n(\%)$ & & & 0.30 \\
\hline Male & $99(73 \%)$ & $37(27 \%)$ & \\
\hline Female & $44(80 \%)$ & $11(20 \%)$ & \\
\hline Age, mean years (SD) & $41.8(17.4)$ & $35.1(16.8)$ & $0.024 *$ \\
\hline Multi-trauma, $n(\%)$ & & & $<0.001^{*}$ \\
\hline No & $123(81 \%)$ & $29(19 \%)$ & \\
\hline Yes & $20(51 \%)$ & $19(49 \%)$ & \\
\hline Trauma mechanism, $n(\%)$ & & & $<0.001^{*}$ \\
\hline Low energy & $94(85 \%)$ & $17(15 \%)$ & \\
\hline High energy & $49(61 \%)$ & $31(39 \%)$ & \\
\hline ASA-PS, $n(\%)$ & & & 0.36 \\
\hline ASA I & $71(77 \%)$ & $21(23 \%)$ & \\
\hline ASA II & $44(69 \%)$ & $20(31 \%)$ & \\
\hline ASA III-IV & $28(80 \%)$ & $7(20 \%)$ & \\
\hline \multicolumn{4}{|l|}{ Open fracture, $n(\%)$} \\
\hline No & $105(82 \%)$ & $23(18 \%)$ & $0.001 *$ \\
\hline Yes & $38(60 \%)$ & $25(40 \%)$ & \\
\hline AO/OTA type, $n(\%)$ & & & $0.003^{*}$ \\
\hline $42 \mathrm{~A} 1-3$ & $98(82 \%)$ & $21(18 \%)$ & \\
\hline $42 \mathrm{~B} 1-3$ & $30(68 \%)$ & $14(32 \%)$ & \\
\hline $42 \mathrm{C} 1-3$ & $15(54 \%)$ & $13(46 \%)$ & \\
\hline Location, $n(\%)$ & & & 0.16 \\
\hline Proximal & $3(50 \%)$ & $3(50 \%)$ & \\
\hline Middle & $43(74 \%)$ & $15(26 \%)$ & \\
\hline Distal & $91(78 \%)$ & $25(22 \%)$ & \\
\hline Segmental & $6(55 \%)$ & $5(45 \%)$ & \\
\hline Fibula fracture, $n(\%)$ & & & 0.39 \\
\hline No & $22(81 \%)$ & $5(19 \%)$ & \\
\hline Yes & $121(74 \%)$ & $43(26 \%)$ & \\
\hline$>2$ proximal screws, $n(\%)$ & & & $0.017 *$ \\
\hline No & $135(77 \%)$ & $40(23 \%)$ & \\
\hline Yes & $8(50 \%)$ & $8(50 \%)$ & \\
\hline$>2$ distal screws, $n(\%)$ & & & 0.86 \\
\hline No & $109(75 \%)$ & $36(25 \%)$ & \\
\hline Yes & $34(74 \%)$ & $12(26 \%)$ & \\
\hline Day of operation, $n(\%)$ & & & $0.012 *$ \\
\hline Weekday & $88(69 \%)$ & $39(31 \%)$ & \\
\hline Weekend & $55(86 \%)$ & $9(14 \%)$ & \\
\hline After-hours surgery, $n(\%)$ & & & 0.86 \\
\hline No & $109(75 \%)$ & $36(25 \%)$ & \\
\hline Yes & $34(74 \%)$ & $12(26 \%)$ & \\
\hline Level surgeon, $n(\%)$ & & & 0.68 \\
\hline Consultant & $51(72 \%)$ & $20(28 \%)$ & \\
\hline Fellow & $51(75 \%)$ & $17(25 \%)$ & \\
\hline Registrar & $41(79 \%)$ & $11(21 \%)$ & \\
\hline
\end{tabular}

*Binary logistic regression or $\chi^{2}$ test was significant at $P<0.05$
Table 5 Multivariable logistic regression analysis subsequent surgery fracture and wound healing

\begin{tabular}{llc}
\hline Variable & $\begin{array}{l}\text { Odds ratio (95\% confi- } \\
\text { dence interval) }\end{array}$ & $P$ value \\
\hline Age & $0.96(0.94-0.99)^{\mathrm{a}}$ & $<0.01$ \\
Multi-trauma & $3.20(1.42-7.22)$ & $<0.01$ \\
Open fracture & $4.14(1.89-9.05)$ & $<0.001$ \\
Surgery on weekdays & $2.96(1.22-7.17)$ & 0.02 \\
\hline
\end{tabular}

${ }^{\text {a } O d d s ~ r a t i o ~ p e r ~ y e a r ~ i n c r e a s e ~ i n ~ a g e ~}$

Although we identified several predictors for subsequent surgery for fracture and wound healing, it remains difficult to extrapolate these findings to the individual patient: we present average results of an 'extrapolated study population'. Moreover, these predictors have not been validated [29]. In orthopaedic surgery, various studies have recently been published that use a streamlined method for developing, validating and deploying prediction models [30, 31]. The use of machine learning algorithms in these studies furthermore may allow for identifying nonlinear relations between variables [32]. Applying such methods could potentially aid in developing, validating and deploying a more practical prediction model to estimate the risk of subsequent surgery in individual patients with TSFs. This may require larger datasets and could be subject of future studies in our era of personalized care.

The current study could not identify any causal predictors of subsequent surgery for removal of implant. This might mostly be determined by type of implant used and local experiences and protocol. Given the high rate of these surgeries, future studies should aim to assess whether there are any other variables associated with these procedures. Identifying such variables may help modifying treatment in order to decrease the rate of these procedures.

In conclusion, nearly one-in-two patients treated with IMN for TSFs will undergo an additional surgical procedure. Approximately half of these procedures are required for wound and fracture healing, whilst the remaining half are discretionarily performed to remove symptomatic screws or nails. Age, open fractures and multi-trauma were independent predictors of the former, whilst a higher rate of symptomatic implant removal was seen in ASA I patients. 
Table 6 Bivariable analysis of patient, trauma, fracture and treatment characteristics and subsequent surgery for symptomatic screws or nail

\begin{tabular}{|c|c|c|c|}
\hline \multirow[t]{2}{*}{ Variable } & \multicolumn{2}{|c|}{$\begin{array}{l}\text { Surgery symptomatic } \\
\text { screws or nail }\end{array}$} & \multirow[t]{2}{*}{$P$ value } \\
\hline & No & Yes & \\
\hline Gender, $n(\%)$ & & & 0.63 \\
\hline Male & $107(79 \%)$ & $29(21 \%)$ & \\
\hline Female & $45(82 \%)$ & $10(18 \%)$ & \\
\hline Age, mean years (SD) & $41.1(18.1)$ & $36.1(14.3)$ & 0.11 \\
\hline Multi-trauma, $n(\%)$ & & & 0.38 \\
\hline No & $119(78 \%)$ & $33(22 \%)$ & \\
\hline Yes & $33(85 \%)$ & $6(15 \%)$ & \\
\hline Trauma mechanism, $n(\%)$ & & & 0.90 \\
\hline Low energy & $88(79 \%)$ & $23(21 \%)$ & \\
\hline High energy & $64(80 \%)$ & $16(20 \%)$ & \\
\hline ASA-PS, $n(\%)$ & & & $0.01 *$ \\
\hline ASA I & $65(71 \%)$ & $27(29 \%)$ & \\
\hline ASA II & $57(89 \%)$ & $7(11 \%)$ & \\
\hline ASA III-IV & $30(86 \%)$ & $5(14 \%)$ & \\
\hline \multicolumn{4}{|l|}{ Open fracture, $n(\%)$} \\
\hline No & $97(76 \%)$ & $31(24 \%)$ & 0.06 \\
\hline Yes & $55(87 \%)$ & $8(13 \%)$ & \\
\hline AO/OTA-type, $n(\%)$ & & & 0.99 \\
\hline $42 \mathrm{~A} 1-3$ & $95(80 \%)$ & $24(20 \%)$ & \\
\hline $42 \mathrm{~B} 1-3$ & $35(80 \%)$ & $9(20 \%)$ & \\
\hline $42 \mathrm{C} 1-3$ & $22(79 \%)$ & $6(21 \%)$ & \\
\hline Location, $n(\%)$ & & & 0.55 \\
\hline Proximal & $6(100 \%)$ & $0(0 \%)$ & \\
\hline Middle & $44(76 \%)$ & $14(24 \%)$ & \\
\hline Distal & $93(80 \%)$ & $23(20 \%)$ & \\
\hline Segmental & $9(82 \%)$ & $2(18 \%)$ & \\
\hline Fibula fracture, $n(\%)$ & & & 0.20 \\
\hline No & $19(70 \%)$ & $8(30 \%)$ & \\
\hline Yes & $133(81 \%)$ & $31(19 \%)$ & \\
\hline$>2$ proximal screws, $n(\%)$ & & & 0.63 \\
\hline No & $140(80 \%)$ & $35(20 \%)$ & \\
\hline Yes & $12(75 \%)$ & $4(25 \%)$ & \\
\hline$>2$ distal screws, $n(\%)$ & & & 0.50 \\
\hline No & $117(81 \%)$ & $28(19 \%)$ & \\
\hline Yes & $35(76 \%)$ & $11(24 \%)$ & \\
\hline Surgery in weekend, $n(\%)$ & & & 0.46 \\
\hline No & $103(81 \%)$ & $24(19 \%)$ & \\
\hline Yes & $49(77 \%)$ & $15(23 \%)$ & \\
\hline After-hours surgery, $n(\%)$ & & & 0.87 \\
\hline No & $115(79 \%)$ & $30(21 \%)$ & \\
\hline Yes & $37(80 \%)$ & $9(20 \%)$ & \\
\hline Level surgeon, $n(\%)$ & & & 0.55 \\
\hline Consultant & $59(83 \%)$ & $12(17 \%)$ & \\
\hline Fellow & $54(79 \%)$ & $14(21 \%)$ & \\
\hline Registrar & 39 (75\%) & $13(25 \%)$ & \\
\hline
\end{tabular}

$* \chi^{2}$ test was significant at $P<0.05$
Table 7 Bivariable analysis of tibiofibular screw penetration and subsequent surgery for screw removal

\begin{tabular}{llrll}
\hline Tibiofibular screw penetration & Total & \multicolumn{2}{l}{ Screw removal } & \multirow{2}{*}{$P$ value } \\
\cline { 3 - 4 } & & \multicolumn{1}{l}{ Yes } & \\
\hline Proximal, $n(\%)$ & & & & \\
$\quad$ No screw penetration & 61 & $7(11 \%)$ & $54(89 \%)$ & 0.51 \\
$\quad$ Screw penetration & 44 & $7(16 \%)$ & $37(84 \%)$ & \\
Distal, $n(\%)$ & & & & \\
$\quad$ No screw penetration & 63 & $10(16 \%)$ & $53(84 \%)$ & 0.57 \\
$\quad$ Screw penetration & 42 & $5(12 \%)$ & $37(88 \%)$ & \\
\hline
\end{tabular}

Acknowledgements Laurent Hendrickx has received unrestricted grants from the Marti-Keuning Eckhardt Foundation, Traumaplatform, and from the Prins Bernhard Cultuurfonds.

Author contributions All authors have made substantial contributions to the following: (1) the conception and design of the study, acquisition of data, analysis of data or interpretation of data and (2) drafting the article or revising it critically for important intellectual content.

Availability of data and material Not applicable.

\section{Compliance with ethical standards}

Conflict of interest The authors, their immediate relatives and any research foundations with which they are affiliated have not received any financial payments or other benefits from any commercial entity related to the subject of this article. Ruurd Jaarsma has given paid presentations not related to this topic for Smith \& Nephew and DePuy Synthes.

Ethical approval In accordance with the Declaration of Helsinki, our institutional review board granted approval for this study (Reference number: AUD/19/SAC/250).

Code availability Not applicable.

Consent to participate Not applicable.

Consent for publication Not applicable.

Open Access This article is licensed under a Creative Commons Attribution 4.0 International License, which permits use, sharing, adaptation, distribution and reproduction in any medium or format, as long as you give appropriate credit to the original author(s) and the source, provide a link to the Creative Commons licence, and indicate if changes were made. The images or other third party material in this article are included in the article's Creative Commons licence, unless indicated otherwise in a credit line to the material. If material is not included in the article's Creative Commons licence and your intended use is not permitted by statutory regulation or exceeds the permitted use, you will need to obtain permission directly from the copyright holder. To view a copy of this licence, visit http://creativecommons.org/licenses/by/4.0/. 


\section{References}

1. Larsen P, Elsoe R, Hansen SH et al (2015) Incidence and epidemiology of tibial shaft fractures. Injury 46:746-750

2. Tornetta P III, Ricci W, McQueen MM et al (2019) Rockwood and Green's fractures in adults. Lippincott Williams \& Wilkins, Philadelphia

3. Stavrou PZ, Ciriello V, Theocharakis S et al (2016) Prevalence and risk factors for re-interventions following reamed intramedullary tibia nailing. Injury 47(Suppl 7):S49-S52

4. Fong K, Truong V, Foote CJ et al (2013) Predictors of nonunion and reoperation in patients with fractures of the tibia: an observational study. BMC Musculoskelet Disord 14:103

5. Bhandari M, Tornetta P 3rd, Sprague S et al (2003) Predictors of reoperation following operative management of fractures of the tibial shaft. J Orthop Trauma 17:353-361

6. Harris I, Lyons M (2005) Reoperation rate in diaphyseal tibia fractures. ANZ J Surg 75:1041-1044

7. Vallier HA, Le TT, Bedi A (2008) Radiographic and clinical comparisons of distal tibia shaft fractures $(4$ to $11 \mathrm{~cm}$ proximal to the plafond): plating versus intramedullary nailing. J Orthop Trauma 22:307-311

8. Leliveld MS, Verhofstad MHJ (2012) Injury to the infrapatellar branch of the saphenous nerve, a possible cause for anterior knee pain after tibial nailing? Injury 43:779-783

9. Sidky A, Buckley RE (2008) Hardware removal after tibial fracture has healed. Can J Surg 51:263-268

10. Weil YA, Gardner MJ, Boraiah S et al (2009) Anterior knee pain following the lateral parapatellar approach for tibial nailing. Arch Orthop Trauma Surg 129:773-777

11. Ricci WM, Gallagher B, Brandt A et al (2009) Is after-hours orthopaedic surgery associated with adverse outcomes? A prospective comparative study. J Bone Joint Surg Am 91:2067-2072

12. Hendrickx LAM, Virgin J, van den Bekerom MPJ et al (2020) Complications and subsequent surgery after intra-medullary nailing for tibial shaft fractures: review of 8110 patients. Injury. https://doi.org/10.1016/j.injury.2020.04.021

13. Busam ML, Esther RJ, Obremskey WT (2006) Hardware removal: indications and expectations. J Am Acad Orthop Surg 14:113-120

14. Reith G, Schmitz-Greven V, Hensel KO et al (2015) Metal implant removal: benefits and drawbacks-a patient survey. BMC Surg 15:96

15. Abouleish AE, Leib ML, Cohen NH (2015) ASA provides examples to each ASA physical status class. ASA Monitor 79(6):38-49

16. Cain ME, Hendrickx LAM, Bleeker NJ et al (2020) Prevalence of rotational malalignment after intramedullary nailing of tibial shaft fractures: can we reliably use the contralateral uninjured side as the reference standard? J Bone Joint Surg Am. https:// doi.org/10.2106/JBJS.19.00731

17. Cain ME, Doornberg JN, Duit R et al (2018) High incidence of screw penetration in the proximal and distal tibiofibular joints after intramedullary nailing of tibial fractures-a prospective cohort and mapping study. Injury 49:871-876

18. Zelle BA, Buttacavoli FA, Shroff JB, Stirton JB (2015) Loss of follow-up in orthopaedic trauma: who is getting lost to follow-up? J Orthop Trauma 29:510-515
19. Vallier HA, Cureton BA, Patterson BM (2011) Randomized, prospective comparison of plate versus intramedullary nail fixation for distal tibia shaft fractures. J Orthop Trauma 25:736-741

20. O'Halloran K, Coale M, Costales T et al (2016) Will my tibial fracture heal? Predicting nonunion at the time of definitive fixation based on commonly available variables. Clin Orthop Relat Res 474:1385-1395

21. Dailey HL, Wu KA, Wu P-S et al (2018) Tibial fracture nonunion and time to healing after reamed intramedullary nailing: risk factors based on a single-center review of 1003 patients. J Orthop Trauma 32:e263-e269

22. Gaebler C, Berger U, Schandelmaier P et al (2001) Rates and odds ratios for complications in closed and open tibial fractures treated with unreamed, small diameter tibial nails: a multicenter analysis of 467 cases. J Orthop Trauma 15:415-423

23. Metsemakers W-J, Handojo K, Reynders P et al (2015) Individual risk factors for deep infection and compromised fracture healing after intramedullary nailing of tibial shaft fractures: a single centre experience of 480 patients. Injury 46:740-745

24. Leliveld MS, Polinder S, Panneman MJM et al (2019) Epidemiologic trends for isolated tibia shaft fracture admissions in The Netherlands between 1991 and 2012. Eur J Trauma Emerg Surg. https://doi.org/10.1007/s00068-018-01072-3

25. Kostis WJ, Demissie K, Marcella SW et al (2007) Weekend versus weekday admission and mortality from myocardial infarction. $\mathrm{N}$ Engl J Med 356:1099-1109

26. Bell CM, Redelmeier DA (2001) Mortality among patients admitted to hospitals on weekends as compared with weekdays. N Engl J Med 345:663-668

27. Honeyford K, Cecil E, Lo M et al (2018) The weekend effect: does hospital mortality differ by day of the week? A systematic review and meta-analysis. BMC Health Serv Res 18:870

28. Labronici PJ, Santos Pires RE, Franco JS et al (2011) Recommendations for avoiding knee pain after intramedullary nailing of tibial shaft fractures. Patient Saf Surg 5:31

29. Steyerberg EW, Vergouwe Y (2014) Towards better clinical prediction models: seven steps for development and an ABCD for validation. Eur Heart J 35:1925-1931

30. Thio QCBS, Karhade AV, Ogink PT et al (2018) Can machinelearning techniques be used for 5-year survival prediction of patients with chondrosarcoma? Clin Orthop Relat Res 476:2040-2048

31. Hendrickx LAM, Sobol GL, Langerhuizen D et al (2019) A machine learning algorithm to predict the probability of (occult) posterior malleolar fractures associated with tibial shaft fractures to guide "malleolus first" fixation. J Orthop Trauma. https://doi. org/10.1097/BOT.0000000000001663

32. Bertsimas D, Dunn J, Velmahos GC, Kaafarani HMA (2018) Surgical risk is not linear: derivation and validation of a novel, user-friendly, and machine-learning-based Predictive OpTimal Trees in Emergency Surgery Risk (POTTER) calculator. Ann Surg 268:574-583

Publisher's Note Springer Nature remains neutral with regard to jurisdictional claims in published maps and institutional affiliations. 